This item was submitted to Loughborough's Research Repository by the author.

Items in Figshare are protected by copyright, with all rights reserved, unless otherwise indicated.

\title{
Control of noise-induced oscillations by delayed feedback
}

PLEASE CITE THE PUBLISHED VERSION

LICENCE

CC BY-NC-ND 4.0

\section{REPOSITORY RECORD}

Balanov, Alexander G., Natalia B. Janson, and E. Scholl. 2019. "Control of Noise-induced Oscillations by Delayed Feedback”. figshare. https://hdl.handle.net/2134/193. 


\title{
Control of noise-induced oscillations by delayed feedback
}

\author{
A.G. Balanov ${ }^{\text {a }}$, N.B. Janson ${ }^{\text {b }}$, E. Schöll ${ }^{\mathrm{a}}$ \\ ${ }^{a}$ Institut für Theoretische Physik, Technische Universität Berlin, Hardenbergstraße \\ 36, D-10623 Berlin, Germany \\ ${ }^{\mathrm{b}}$ Department of Mathematical Sciences, Loughborough University, \\ Loughborough,Leicestershire LE11 3TU, UK
}

\begin{abstract}
We propose a method to control noise-induced motion, based on using delayed feedback in the form of the difference between the delayed and the current states of the system. The method is applied to two different types of systems, namely, a selfoscillator near Andronov-Hopf bifurcation and a threshold system. In both cases we demonstrate that by variation of time delay one can effectively control coherence and timescales of stochastic oscillations. The entrainment of the basic period of oscillations by time delay is discovered. We give explanations of the phenomena observed and provide a theory for the system near bifurcation.
\end{abstract}

Key words: noise-induced oscillations, control, delayed feedback PACS: 05.40.-a, 05.45.-a, 02.30.Yy

\section{Introduction}

Control of complex irregular motion is one of the central problems of applied nonlinear dynamics. As applied to oscillations, control usually means an adjustment of essential oscillation properties: stability, coherence, timescales, etc. in a desirable manner by imposing a small perturbation on the system. Within the last decades a substantial progress has been achieved in the development of methods for the control of dynamical chaos [1-3], where the complexity has a deterministic origin, and appears whenever the evolution of a dynamical system depends sensitively on initial conditions. Originally, control of chaotic motion assumed turning it into a regular, predictable one, by influencing the system in an intelligent way. Most techniques for controlling chaos exploit the idea of stabilization of unstable periodic orbits that are supposed to exist in the systems to be controlled. There are three general approaches: a continuous 
external perturbation [4], a time-discrete conditioned perturbation [5], and a feedback loop in the form of the difference between the current state of the system and its state some $\tau$ time units ago [6].

Random fluctuations can be another source of irregularity in motion. For a long time it has been thought that random noise alone can only bring disorder into the system, while being unable to produce any ordered motion. However, nowadays this opinion has undergone serious revision. It has been shown that noise influencing a nonlinear system can enhance the already ordered motion, and sometimes even give rise to quite a coherent dynamics. Striking examples are stochastic resonance $[7,8]$, when noise added to a nonlinear system enhances its response to a useful signal, and coherence resonance when noise applied to an excitable system generates oscillations similar to selfsustained ones, whose coherence depends resonantly on noise intensity $[9,10]$. Nonlinear systems that do not oscillate autonomously but demonstrate coherence resonance are often called coherence resonance oscillators. These kinds of phenomena seem to be prominent in many areas of science, e.g. physics, biology, chemistry etc. [11-16]. With this, in contrast to deterministic chaos, the control of noise-induced phenomena is still an open problem. Recently, a number of methods were suggested for the control of stochastic resonance $[17,18]$ and of self-oscillations in the presence of noise [19]. In [20] an external periodic force was proposed for the control of noise-induced oscillations in a pendulum with a randomly vibrating suspension axis. All methods mentioned above assume the presence of deterministic self-oscillating components in the dynamics, and aim either to enhance the latter component or to exploit the external deterministic oscillating force to manipulate the regularity of motion. In this paper we suggest a passive self-adaptive method for the control of oscillations induced merely by noise. We show that the delayed feedback in the form used previously for the control of deterministic chaos [6] including spatio-temporal patterns [21-23] allows one to effectively manipulate the properties of coherence resonance oscillations, that includes the adjustment of their timescales.

The paper has the following structure: after the Introduction, in Section 2 the motivation for the proposed control method is given, and in Section 3 the effect of delayed-feedback on the two different types of systems is described. Section 4 presents the Conclusions.

\section{Motivation for the approach proposed}

As mentioned above, the idea of applying a delayed feedback in order to control chaotic motion is based on the fact that a chaotic attractor contains a countable set of unstable periodic orbits embedded into it. Then, using, for 
instance, the feedback $F(t)$ in the form:

$$
F(t)=K(s(t-\tau)-s(t))
$$

where $s(t)$ is the signal coming from the system, $K$ is the feedback strength, and $\tau$ is time delay being equal to the period $T_{0}$ of a selected orbit, can make this orbit stable [6]. Recently, the influence of the feedback as in [6] on a deterministically chaotic system was studied for a small range of $\tau$ in the vicinity of $T_{0}$ [24], and the ability of the feedback to change the period of a stabilized orbit was demonstrated.

In a coherence resonance oscillator there are no deterministic orbits. However, noise makes such a system oscillate in quite a regular manner, giving rise to phase portraits that are reminiscent of smeared-out limit cycles (see Fig. 1 for an example). Remarkably, such stochastic oscillations were shown to possess the fundamental property of deterministic self-oscillations, namely, the ability to synchronize [25]. The analogies with self-sustained systems gave rise to the term "stochastic limit cycle" [26] to refer to the coherent motion in the phase space that has arisen thanks to external random force. The "stochastic cycle" has a shape that is characteristic of the given system, and a period that is jointly defined by the structure of the phase space and by noise. Using the analogy between a stochastic limit cycle and a deterministic periodic orbit, one can suppose that a delayed feedback applied to the former would produce an effect similar to the one when applied to the latter, provided that the time delay is equal or close to the basic or mean period [27] of the noise-induced motion. Namely, it should suppress large deviations from the "stochastic cycle" and thus enhance the regularity of oscillations. The control force can be expected to be minimal if the time delay $\tau$ is equal to the period of the "stochastic cycle". However, it should not vanish since there is no deterministic periodic orbit in the system. In order to check this, we apply delayed feedback (1) to two different types of systems, namely to a dynamical system near bifurcation and to a threshold excitable system. A Van der Pol oscillator below Andronov-Hopf (AH) bifurcation and a simplified FitzHugh-Nagumo system are considered as prototype examples of the former and the latter classes, respectively.

\section{$3 \quad$ Systems studied and results}

\subsection{Van der Pol oscillator below the Andronov-Hopf (AH) bifurcation.}

The equations describing a Van der Pol oscillator with a delayed feedback loop in the presence of noise read: 

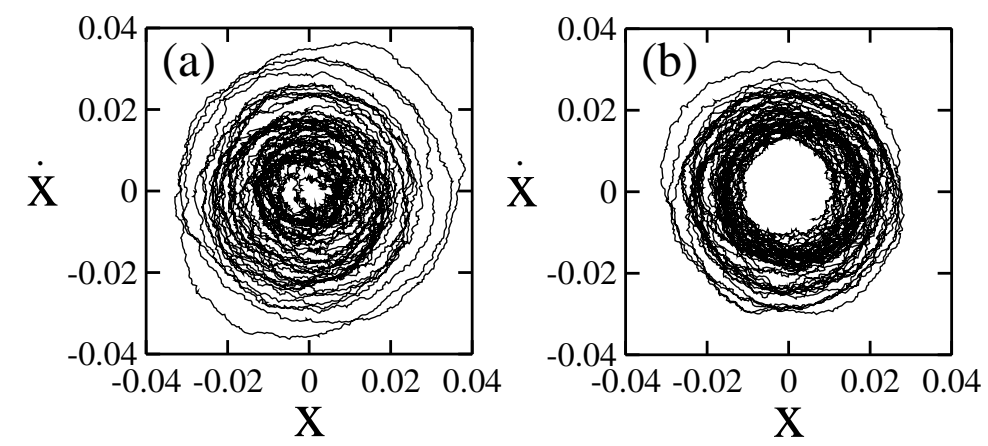

Fig. 1. Phase portraits of noise-induced motion in Van der Pol system at $\omega_{0}=1$, $\varepsilon=-0.01, D=0.003$ : (a) without feedback $K=0$; (b) with feedback $K=0.2$, $\tau=T_{0}$.

$$
\ddot{x}-\left(\varepsilon-x^{2}\right) \dot{x}+\omega_{0}^{2} x=K(\dot{x}(t-\tau)-\dot{x})+D \xi(t) .
$$

Here $\omega_{0}$ defines the natural period of oscillations without feedback, $K$ represents the delayed feedback strength, $\tau$ is time delay, $\xi(t)$ is Gaussian white noise with zero mean and intensity defined by the parameter $D$. The nonlinearity parameter $\varepsilon$ governs the dynamics of the system. At $K=0, D=0$ and a negative $\varepsilon$, the system does not exhibit self-oscillations. At $\varepsilon=0$ the $\mathrm{AH}$ bifurcation occurs, after which at $\varepsilon>0$ a limit cycle exists in the phase space of the system. We fix $\varepsilon$ at a value -0.01 slightly below the AH bifurcation. In this case the only attractor in the system is a stable focus. However, inclusion of noise $(D>0)$ into the system can evoke motion in the phase space that in many respects resembles noisy oscillations with basic period $T_{0} \approx 2 \pi / \omega_{0}$ (see Fig. 1(a)). To quantify the regularity of these oscillations one needs to introduce a certain order parameter, for example, correlation time $t_{c o r}$ as

$$
t_{c o r}=\frac{1}{\sigma^{2}} \int_{0}^{\infty}|\Psi(s)| d s,
$$

where $\Psi(s)=\langle(\dot{x}(t-s)-\langle\dot{x}\rangle)(\dot{x}(t)-\langle\dot{x}\rangle)\rangle$ is the autocorrelation function of signal $\dot{x}(t)$ and $\sigma^{2}$ is its variance. In Fig. 2 the dependence of $t_{c o r}$ on the noise intensity is shown by grey circles. From Fig. 2 it is seen that the regularity of oscillations is larger for smaller noise $D$. Noise-induced motion in the systems near bifurcations was considered in $[28,29]$. Note that, as our calculations have shown, in the Van der Pol system below AH bifurcation the basic period of noise-induced oscillations $T_{0}$ almost does not change with variation of noise intensity.

To check if the delayed feedback can enhance the regularity of noise-induced motion, we set $K=0.2$, and fix $\tau=6.1728$ at a value close to the basic period $T_{0}$ of stochastic oscillations without feedback. In Fig. 1(b) the phase portrait of noise-induced motion under control for $D=0.003$ is shown. In comparison with Fig 1(a) obtained for the same value of $D$ and $K=0$, the 


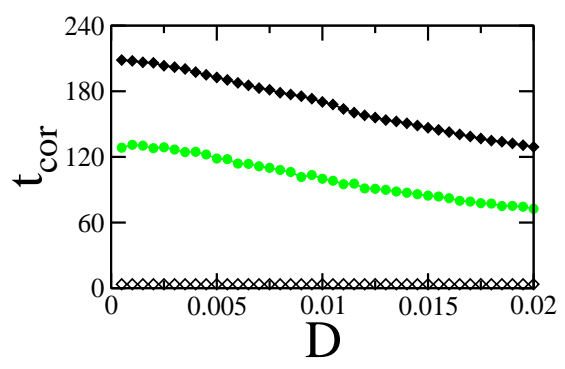

Fig. 2. Van der Pol system. Correlation time $t_{\text {cor }}$ vs noise intensity $D$ : without delayed feedback, $K=0$ (grey circles), and with delayed feedback with $K=0.2$ at $\tau=T_{0}$ (black diamonds) and at $\tau \neq T_{0}$ (white diamonds). Exact values of $\tau$ are given in the text.

obvious ordering takes place which is reflected in a more regular rotation of the phase trajectory around the fixed point at the origin. It is also confirmed by estimation of correlation time $t_{c o r}$ vs $D$ for $K=0.2$ which is shown in Fig. 2 by black diamonds. A remarkable increase of coherence is observed for all values of noise intensity. Thus, we can conclude that delayed feedback can make noise-induced oscillations more ordered.

We note that increase of coherence of noise-induced motion (black diamonds in Fig. 2) is observed if $\tau$ is close to $T_{0}$ or its multiples. However, if $\tau$ is different from that, the coherence can be decreased substantially by the feedback: white diamonds in Fig. 2 show $t_{\text {cor }}$ vs $D$ for $\tau=3$.3. At this value of $\tau$, the coherence is, on the contrary, deteriorated.

After our ability to change deliberately the coherence of noise-induced motion with the help of a feedback is demonstrated, consider if (and how) we can manipulate the timescales of the process. The control parameter that is expected to effect the system timescales is time delay $\tau$. To assess the effect of the value of $\tau$ on the performance of the feedback, we calculate the Fourier Power Spectrum (FPS) of stochastic oscillations for a range of $\tau$. Below we will refer to FPS as to simply "spectrum" for brevity. For $K=0.2$ and $D=0.003$ the spectra are shown in Fig. 3. Since we are interested in comparing the timescales of oscillations with the time interval $\tau$ rather than with some frequency $f$, the spectra in the Fig. 3 are presented in terms of periods $T=1 / f$. Without the feedback $(\tau=0)$, the spectrum has only one pronounced peak with period $T_{1}$ equal to $T_{0}$. However, as $\tau$ increases from zero more peaks appear in the spectrum. For a better illustration of spectral properties, we extract all spectrum peaks, order them with respect to decreasing heights, denote their periods as $T_{i}, i=1,2, \ldots$ and plot $T_{i}$ vs $\tau$ in Fig. 4(d). The basic period $T_{1}$ is shown by light-grey circles, while other periods $T_{i}, i=2,3, \ldots$ are denoted by grey diamonds. We can make three important observations: (i) the periods $T_{i}$ of all peaks change with $\tau$, (ii) the evolution of $T_{1}$ has almost piecewise linear character; (iii) the heights and the widths of spectrum peaks vary as $\tau$ changes. The first two observations mean that the delayed 


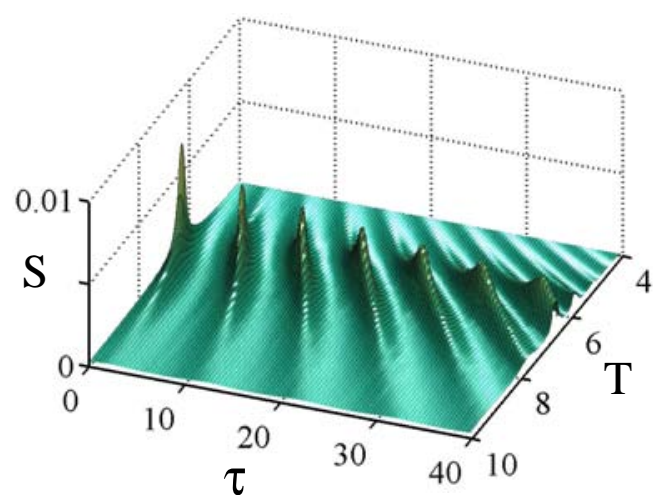

Fig. 3. Spectra of noise-induced oscillations in the Van der Pol system in the presence of delayed feedback for a range of $\tau$ at $D=0.003, K=0.2$

feedback entrains the timescales of noise-induced oscillations: the change in $\tau$ results in an almost proportional change in $T_{i}$. The third observation implies that by varying the delay one can control the regularity of oscillations. As seen from Fig. 4(a), the function $t_{\text {cor }}$ of $\tau$ has an oscillatory character with maxima at $\tau \approx n T_{0}$ and minima $\tau \approx(2 n-1) T_{0} / 2$, where $n$ is an integer. In Fig 4(b) the variance of the control force $F(t)=(\dot{x}(t-\tau)-\dot{x}(t))$ is given as a function of $\tau$ [30]. It is interesting that minima of $\left\langle F^{2}\right\rangle$ correspond to the maxima of $t_{c o r}$. That means that less force is required to control more regular behavior. However, as we expected, in contrast to the deterministic case, this force never vanishes. Explanation of these effects requires a general analysis of Eq. (2), which appears to be a difficult task, since due to the delay the process described by this equation is essentially non-Markovian, and the powerful methods like Fokker-Planck equations suitable for Markovian processes, are not applicable here. At present, the theory of stochastic delay differential equations is under development, and the methods created so far mostly cover linear and very specific cases of nonlinear systems [31-37,19]. However, in our analysis we can exploit the fact that in Eq. (2) the oscillations occur in the vicinity of the fixed point. Hence we assume that the local properties of this point should effect the noise-induced motion. Let us consider a general case of a canonic nonlinear oscillator with time-delayed feedback:

$$
\ddot{x}+f(x, \dot{x})-K(\dot{x}(t-\tau)-\dot{x})=0,
$$

or

$$
\begin{aligned}
& \dot{x}=y \\
& \dot{y}=-f(x, y)+K(y(t-\tau)-y),
\end{aligned}
$$

that describes Eq. (2) with $f(x, \dot{x})=-\left(\varepsilon-x^{2}\right) \dot{x}+\omega_{0}^{2} x$. At $\mathrm{K}=0$, Eq. (4) possesses a single fixed point defined by the equation $f\left(x_{0}, 0\right)=0$. With this, 


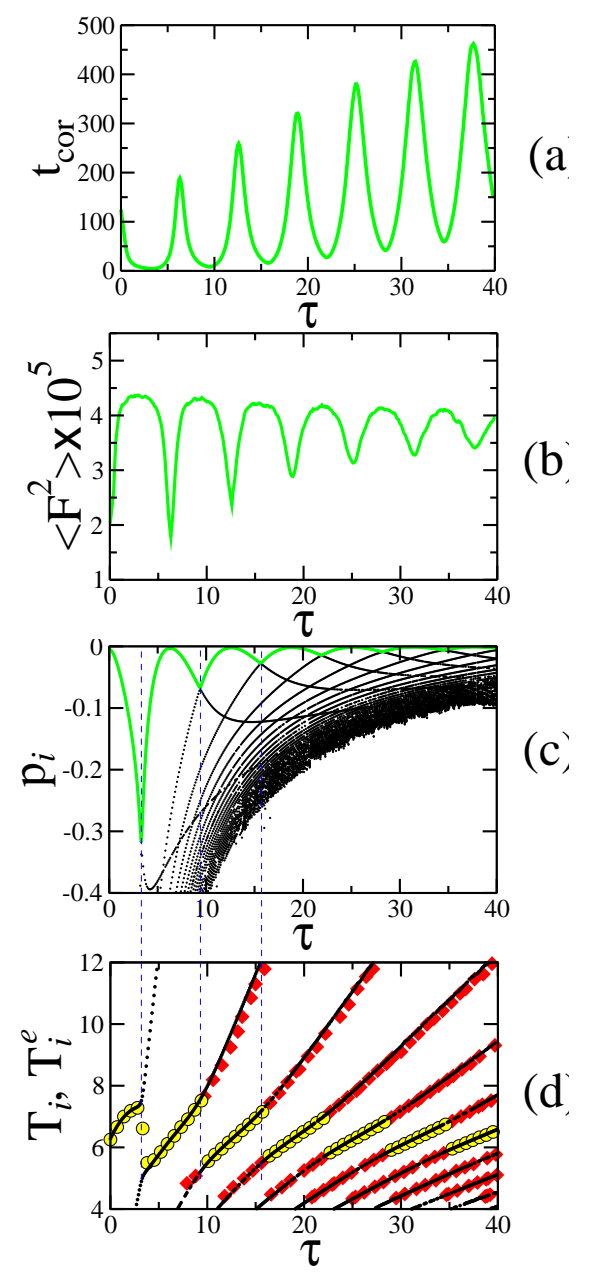

Fig. 4. (a) Correlation time $t_{c o r}$, (b) variance $\left\langle F^{2}\right\rangle$ of control force, (c) real parts $p_{i}, i=1,2, \ldots$ of eigenvalues and (d) spectrum peak periods $T_{i}$ vs $\tau$ at $D=0.003$ and $K=0.2$. In (c) the largest of $p_{i}$ 's, i.e. $p_{1}$, is marked by grey. In (d) light-grey circles - basic period $T_{1}$, grey diamonds - $T_{i}, i=2,3, \ldots$, black dots - eigenperiods $T_{i}^{e}=2 \pi /\left|q_{i}\right|, i=1,2, \ldots$

if

$$
0<\left.\frac{\partial f}{\partial \dot{x}}\right|_{\left(x_{0}, 0\right)}<\left.2 \sqrt{\frac{\partial f}{\partial x}}\right|_{\left(x_{0}, 0\right)},
$$

the fixed point $\left(x_{0}, 0\right)$ is a stable focus. The delayed feedback at $K>0$ does not change the position of the fixed point, however it could influence the local properties of the point that are defined by its eigenvalues. Following the standard routine of linearizing Eq. (4) around the fixed point, the characteristic equation for $\lambda$ is derived:

$$
\lambda^{2}+\left.\lambda \frac{\partial f}{\partial \dot{x}}\right|_{\left(x_{0}, 0\right)}+\left.\frac{\partial f}{\partial x}\right|_{\left(x_{0}, 0\right)}-K \lambda\left(e^{-\lambda \tau}-1\right)=0
$$


Using the ansatz $\lambda=p+i q$ we separate real $(p)$ and imaginary $(q)$ parts of $\lambda$ :

$$
\begin{gathered}
p^{2}-q^{2}+\left.\frac{\partial f}{\partial x}\right|_{\left(x_{0}, 0\right)}+\left.p \frac{\partial f}{\partial \dot{x}}\right|_{\left(x_{0}, 0\right)}+K\left(p-p e^{-p \tau} \cos q \tau-q e^{-p \tau} \sin q \tau\right)=0 \\
2 p q+\left.q \frac{\partial f}{\partial \dot{x}}\right|_{\left(x_{0}, 0\right)}+K\left(q+p e^{-p \tau} \sin q \tau-q e^{-p \tau} \cos q \tau\right)=0
\end{gathered}
$$

Let us analyze the obtained Eqs. (8). First, check if the feedback in the form used might possibly induce the birth of a stable limit cycle via AH bifurcation, thus providing a trivial explanation for the remarkable ordering of oscillations. The condition for AH bifurcation is $p=0, q \neq 0$. Substituting it into the second of Eqs. (8) we obtain:

$$
\cos q \tau=\frac{K+\left.\frac{\partial f}{\partial \dot{x}}\right|_{\left(x_{0}, 0\right)}}{K} .
$$

Condition (6) implies that the right-hand part of Eq. (9) is larger than unity, whereas the left-hand part is always smaller than unity. This contradiction means that an $\mathrm{AH}$ bifurcation does not occur for any $K$ and $\tau$, i.e. no limit cycle is born from the fixed point.

Now, consider if and how $p$ and $q$ are related to the properties of noise-induced oscillations. The real part $p$ defines the stability of the fixed point. At $\tau=0$ the fixed point has two complex-conjugate eigenvalues with negative $p_{1,2}$. As $\tau$ gets positive, the system becomes infinite-dimensional with a countable set of eigenvalues, whose real parts appear from minus infinity and always remain negative. We order all eigenvalues $\lambda_{i}, i=1,2, \ldots$ in descending order of their real parts $p_{i}$, so that $p_{1}$ is the largest one. In Fig. 4(c) $p_{i}$ are given as functions of $\tau . p_{1}$ (highlighted by a grey line) has a pronounced oscillatory character as $\tau$ increases and correlates well with the graph for $t_{c o r}$ in Fig. 4(a). That means that the motion induced by noise is most coherent ( $t_{c o r}$ is the largest) when the fixed point is least stable ( $p_{1}$ is closest to zero).

Each imaginary part $q_{i}$ describes the velocity of rotation of the phase trajectory around the fixed point that is associated with its $i$ th eigenmode. The corresponding eigenperiods $T_{i}^{e}=2 \pi /\left|q_{i}\right|$ are given in Fig. $4(\mathrm{~d})$ by black dots and seem to coincide remarkably with the spectrum peak periods $T_{i}$. To avoid overloading the picture, we do not color-code the period $T_{1}^{e}$ of the least stable eigenmode here, but note that it always coincides with $T_{1}$. Also, the other branches of $T_{i}^{e}$ that are close to the spectral period $T_{i}$ are related to the set of eigenmodes, which are least stable. The latter means that in system (4) with delayed feedback noise excites those eigenmodes which are least stable. The piecewise behavior of $T_{1}$ is due to intersections between different branches of 

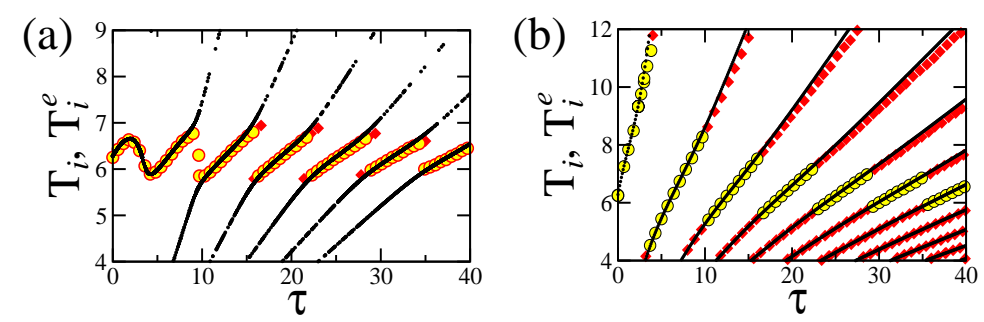

Fig. 5. Evolution of spectral peak periods $T_{i}$ of noise-induced oscillations in Van der Pol system as a function of $\tau$ at $D=0.003$ for different values of $K$ (compare with Fig. 4(d)): (a) $K=0.1$, (b) $K=0.5$. Notation as in Fig. 4(d).

$p_{i}$ in Fig. 4(c): at the point of intersection, another spectral peak becomes the highest one. The coincidence of $T_{i}$ and $T_{i}^{e}$ was also confirmed for other feedback strengths $K$ as illustrated in Fig. 5 .

Interestingly, if $|\varepsilon|<<K$ then from Eq. (9) it follows that $\cos q \tau \approx 1$ and $q \tau \approx 2 \pi n$ with integer $n$. Then the eigenperiod $T_{1}^{e}$ is

$$
T_{1}^{e}=2 \pi /\left|q_{1}\right| \approx \tau / n
$$

Eq. (9) and, consequently, Eq. (10), hold the better, the closer $p_{1}$ is to zero. This explains the almost piecewise-linear behaviour of the basic periods $T_{1}$ in Figs. 4(d) and 5 at large $\tau$, at which the condition $p_{1} \approx 0$ holds more accurately (Fig. 4(c)). Formula (10) also provides the values of $\tau$ corresponding to the maxima of coherence of noise-induced oscillations. As mentioned above, the maximal coherence corresponds to the least stability of the fixed point, that is to the proximity of $p_{1}$ to zero. Then we substitute $p_{1}=0,\left|q_{1}\right|=2 \pi n / \tau$, and $f(x, \dot{x})$ of the Van der Pol system into the first of Eqs. (8), and finally obtain $\tau=2 \pi n / \omega_{0}=n T_{0}$, which agrees well with the numerical results (see Fig. 4(a)).

Now, consider how another parameter of the feedback, namely, the strength $K$, influences the noise-induced oscillations. Fig. 6 illustrates the peak periods $T_{1,2}$ of two spectrum peaks and eigenvalues of the fixed point as functions of $K$. We set $D=0.003$ and analyze two distinct cases of time delay $\tau$. Namely, $\tau=6.1728 \approx T_{0}$ (Fig. 6(a),(c)), and $\tau=3.3$ (Fig. 6(b),(d)), at which the feedback with $K=0.2$ produces the most and the least coherent oscillations, respectively. For $\tau$ almost equal to the basic period $T_{0}$ of uncontrolled oscillations, the increase of $K$ does not change the position of the basic peak with period $T_{1}=T_{0}$ (light-grey cirles in Fig. $6(\mathrm{a})$ ). With this, at $K \approx 0.25$ another peak appears whose period $T_{2}$ (grey diamonds in Fig. 6(a)) monotonically increases with $K$. Eigenperiods $T_{1,2}^{e}$ of the two least stable eigenmodes are shown by black dots in Fig. 6(a); they coincide with reasonable accuracy with $T_{1,2}$, respectively. The slight discrepancy between $T_{2}$ and $T_{2}^{e}$ (upper branches) is probably due to the fact that the estimate of the central period of a wide 

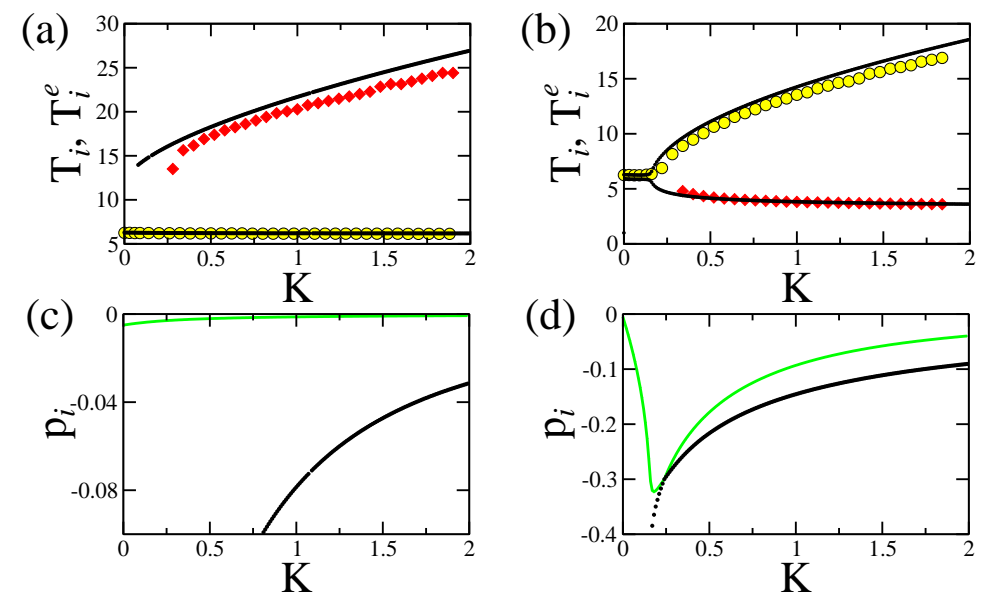

Fig. 6. (a), (b) Spectral peak periods $T_{1,2}$ (circles, diamonds) and eigenperiods $T_{1,2}^{e}$ (dots); (c), (d) the two largest real parts $p_{1,2}$ of eigenvalues of the fixed point in the Van der Pol system vs $K$ at $D=0.003$. (a), (c) $\tau=6.1728$, (b), (d) $\tau=3.3$. Symbols are the same as in Figs. 4 (c) and (d).

and low spectrum peak that is almost hidden in the background introduces some numerical error. If, however, $\tau=3.3, T_{1}$ remains close to $T_{0}$ for a range of $K \lesssim 0.21$. As $K$ is increased further, the basic peak splits into two, with periods $T_{1}$ and $T_{2} . T_{1}$ grows monotonically with $K$, while $T_{2}$, remaining smaller, tends to coincide with $\tau$ for large $K$.

The evolution of heights of the two peaks roughly follows the evolution of the real parts $p_{1,2}$ of the eigenvalues shown in Figs. 6 (c) and (d). Whereas at $\tau=6.1728$ the growth of $K$ leads to increase in both $p_{1}$ and $p_{2}$, which is associated with an enhancement of both eigenmodes (Fig. 6(c)), at $\tau=3.3$, $p_{1,2}$ evolve non-monotonically. As seen from Fig. $6(\mathrm{~d})$, at $K \lesssim 0.21, p_{1}$ (grey lines in Fig. 6(d)) drops, whereas $p_{2}$ (black dots in Fig. 6(d)) increases. This means that as $K$ increases, the mode associated with $T_{0}$ is being gradually suppressed, while the mode with period close to $\tau$ is enhanced by delayed feedback. Starting from $K \approx 0.21$, the suppression of the basic eigenmode stops, and further increase of $K$ enhances both modes.

\subsection{Simplified FitzHugh-Nagumo system.}

Now consider another example of an excitable system, namely, a simplified FitzHugh-Nagumo system which is often treated as a prototype of excitable media like neurons and reaction-diffusion systems:

$$
\mu \frac{d x}{d t}=x-\frac{x^{3}}{3}-y
$$



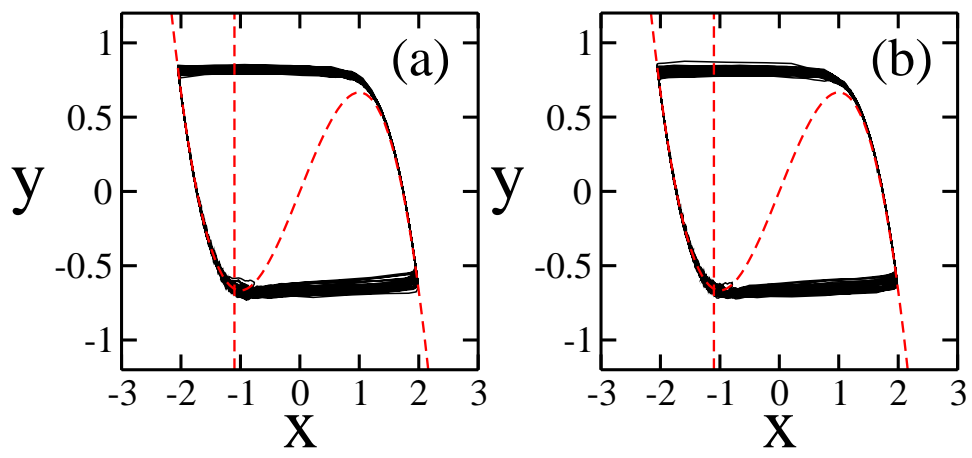

Fig. 7. Phase portraits of noise-induced motion in the FitzHugh-Nagumo system at $D=0.09, \mu=0.01, a=1.1$ : (a) without feedback $K=0$; (b) with feedback $K=0.2, \tau=T_{0}$.

$$
\frac{d y}{d t}=x+a+K(y(t-\tau)-y)+D \xi(t)
$$

We choose the parameters $\mu=0.01$ and $a=1.1$ such that a stable node is the only attractor of the system in the absence of feedback. Here, as in Eq. (2) $\tau$ is a time delay, $K$ is the feedback strength, and $\mathrm{D}$ is the intensity of Gaussian white noise $\xi$ with zero mean. In contrast to the system considered in Section 3.1, the oscillations induced by noise in Eq. (11) have a nonlocal character (see Fig. 7(a)). Null-isoclines (lines defined by $\dot{x}=0$ and $\dot{y}=0$ ) shown in Fig. 7 by dashed lines separate different directions of motion in the phase space. They intersect at the fixed point. The noise-induced motion includes two components: oscillations near the fixed point and long excursions along branches of null-isocline defined by $\dot{x}=0$, i.e. by $y=x-x^{3} / 3$. Because $\mu$ is chosen to be small, the motion between the fixed point and the right-hand branch of the cubic parabola, and between the right-hand maximum of the parabola and left-hand branch, occurs almost instantly, and the associated time contribution can be reasonably neglected. This means that each instantaneous period of oscillations contains two components: time of escape from the vicinity of the fixed point (activation time) and time of motion along the branches of the parabola (excursion time) [38]. To initiate an excursion, the phase trajectory should be kicked out of a certain vicinity of the fixed point, which allows one to treat this kind of excitability as "a threshold excitability". Although Eqs. (11) do not conform to the canonical oscillator Eq. (4), the stability of the fixed point can be checked analytically using the standard approach. It has been shown that delayed feedback does not induce AH bifurcation in Eq. (11). The global character of oscillations renders the approach used for the Van der Pol system in Section 3.1 inappropriate. Therefore, in this Section we present only the results of numerical simulation.

Since the essential component of the oscillations is motion along null-isoclines, on which the noise influence is small, a visual comparison of the phase portraits of the system without control (Fig. 7(a)) and with control (Fig. 7(b)) does not 

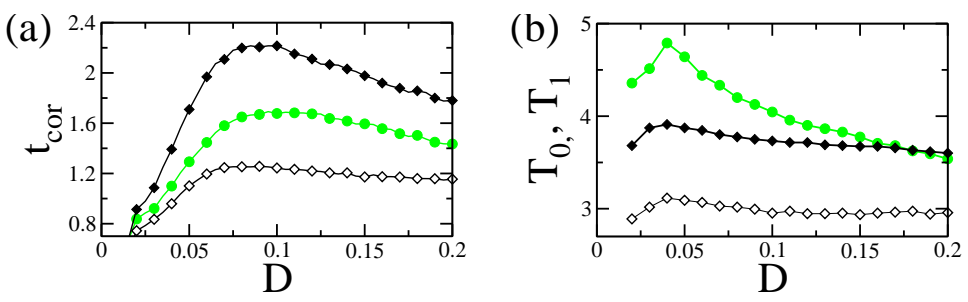

Fig. 8. FitzHugh-Nagumo system. (a) Correlation time $t_{c o r}$ and (b) basic periods of noise-induced oscillations without delayed feedback $K=0$ (grey circles), and with delayed feedback with $K=0.2$ at $\tau=T_{0}$ (black diamonds), and $\tau \neq T_{0}$ (white diamonds) as functions of noise intensity $D$. Exact values of $\tau$ are given in the text.

yield a significant difference. However, the calculation of correlation time $t_{c o r}$ using Eq. (3) reveals that as in an oscillator below AH bifurcation, the delayed feedback can remarkably increase the coherence of oscillations given the time delay $\tau$ is chosen appropriately. Fig. 8(a) shows how $t_{c o r}$ depends on noise intensity $D$ in the absence of feedback, $K=0$ (grey circles), and at $K=0.2$ (black diamonds) with $\tau=4.12694$ close to the basic period $T_{0}$ of uncontrolled oscillations at optimal noise $D=0.09$.

It is clearly seen that the feedback with the given parameters amplifies the regularity of noise-induced motion practically for any value of $D$. The presence of an activation time in the system dynamics makes the timescale of its oscillations quite dependent on noise intensity [38]. In Fig. 8 (b) grey circles show the basic period $T_{0}$ of noise-induced oscillations vs noise-intensity $D$ for $K=0$, while black diamonds show the basic period $T_{1}$ when the control with the above parameters is applied. These graphs show that the delayed feedback reduces the range of variation of the basic oscillation period under the change in noise strength. The latter is a consequence of the fact that the control orders and stabilizes the time intervals between successive excursions. For comparison, we have applied feedback control with $K=0.2$ and a delay time $\tau=3$ that is essentially different from $T_{0}$ (white diamonds in Fig. 8 (a),(b)). With this $\tau$ the coherence of oscillations decreases, the basic period $T_{1}$ shifts closer to $\tau$, and the range of its variation remains approximately the same as under control with $\tau=T_{0}$. Hence, application of delayed feedback to a FitzHugh-Nagumo oscillator can not only change the regularity of noise-induced oscillations, but also make them more robust against variation in noise intensity.

Next, we study how the feedback parameters effect the properties of oscillations. First, consider spectra of oscillations for a range of time delay $\tau$ (Fig. 9). It seems that the effect of delay on a system with "threshold excitability" is in many respects similar to the one on a system near AH bifurcation (compare with Fig. 3). Actually, in both cases the variation of $\tau$ leads to the variation of the number, position, height and width of spectrum peaks. Moreover, like in systems near bifurcation, the entrainment of timescales of 


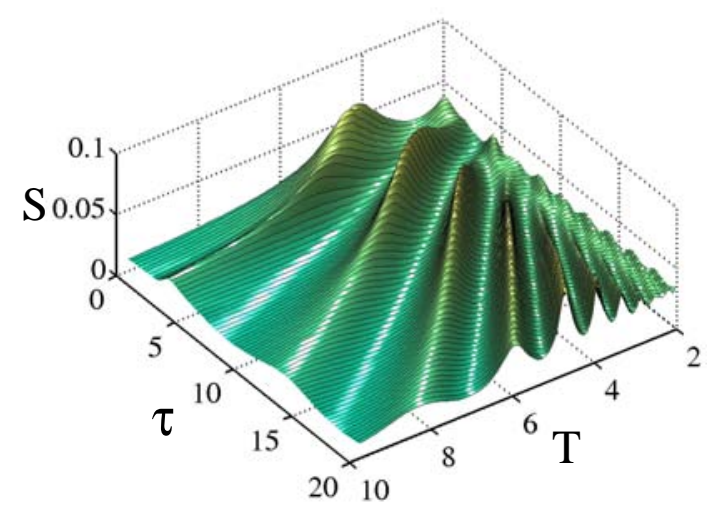

Fig. 9. Spectra of noise-induced oscillations in the FitzHugh-Nagumo system in the presence of delayed feedback for a range of $\tau$ at $D=0.09, K=0.2$

noise-induced oscillations by delayed feedback occurs, which manifests itself in an almost piecewise-linear shift of peak positions as $\tau$ increases (Fig. 10(c)). However, unlike in the Van der Pol system, the spectrum of oscillations in the FitzHugh-Nagumo system contains several pronounced peaks even without control $(\tau=0)$, which makes the effect of control more complicated. Fig. 10 reflects the influence of delay on the oscillation regularity $t_{c o r}$ (a), and on the variance $\left\langle F^{2}\right\rangle$ of the control force $F(\mathrm{~b})$. Both characteristics exhibit oscillatory character. Similarly to the Van der Pol oscillator, the global minimum of the control force in the FitzHugh-Nagumo system is achieved at $\tau$ approximately equal to the basic period $T_{0}$ of oscillations without control $T_{0} \approx 4.12694$. All the other minima of $\left\langle F^{2}\right\rangle$ occur at $\tau$ close to multiples of $T_{0}$. However, the minima of $\left\langle F^{2}\right\rangle$ do not coincide with the maxima of $t_{c o r}$. Comparison with Fig. 10(c) reveals that the maxima of coherence occur when the basic period $T_{1}$ becomes equal to $T_{0}$. The non-coincidence could be due to the presence of two components in the system motion that are affected by the feedback in different ways. Actually, the activation times are most regular at $\tau \approx T_{0}$, that means that a smaller force is required to kick the trajectory out of the vicinity of the fixed point. At the same time, the highest basic peak in the spectrum exists at $\tau \approx 5.1$, at which the oscillations are most coherent on the whole. With this, the shift of spectral peaks as $\tau$ increases is close to linear (Fig. 10(c)) like in the case of the Van der Pol system, that means the entrainment of timescales of stochastic oscillations. Moreover, the presence of entrainment seems not to depend on the feedback strength $K$ in a reasonable range of $K$ (Fig. 11). The increase of $K$ can lead to the enrichment of spectral content of motion, however the linear character of $T_{1}$ vs $\tau$ is not affected. Fig. 12 illustrates how an increase of $K$ can change the spectral peak positions for two distinct values of $\tau$ : (a) for $\tau=4.12694 \approx T_{0}$, and (b) for $\tau=2.9$ at which the global minimum of $t_{\text {cor }}$ occurs (see Fig. 10(a)). At $\tau=4.12694$ the feedback slightly decreases the basic period $T_{1}$ against $T_{0}$ until it reaches about 3.7 at $K \approx 0.2$. As $K$ is increased further, $T_{1}$ slowly increases until it approaches the value of 4 at $K=2$. Several other peaks with smaller periods, which exist 

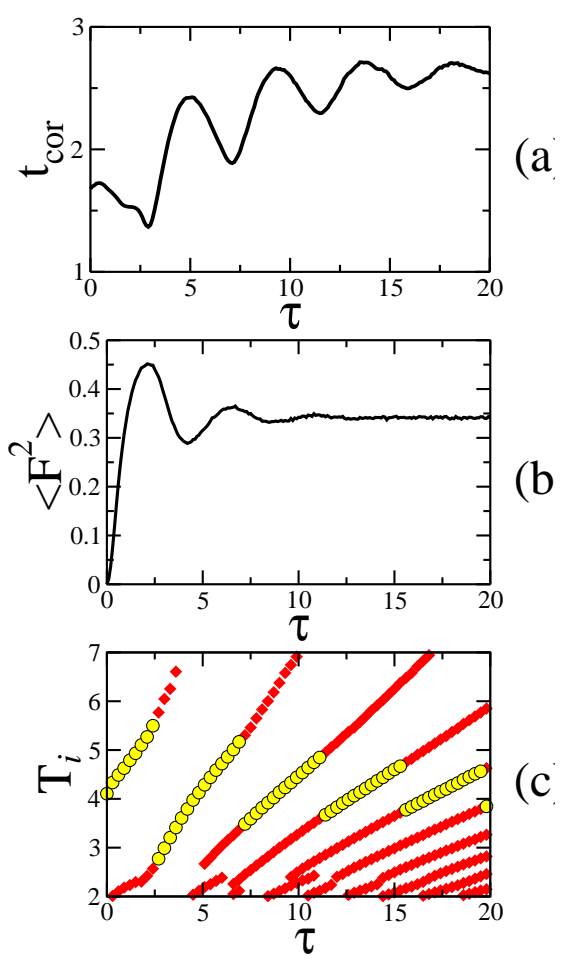

Fig. 10. (a) Correlation time $t_{c o r}$, (b) variance $\left\langle F^{2}\right\rangle$ of control force and (c) peak periods $T_{i}, i=1,2, \ldots$ vs $\tau$ at $D=0.09$ and $K=0.2$.

(a)

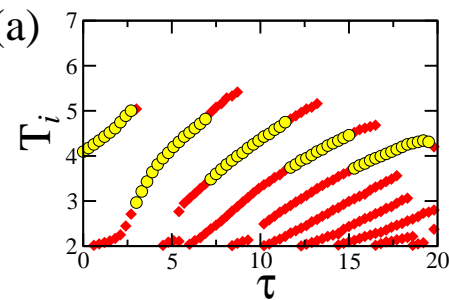

(b)

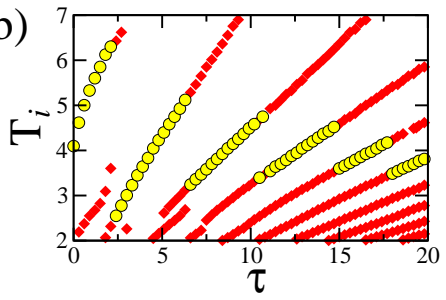

Fig. 11. Evolution of peak periods $T_{i}, i=1,2, \ldots$ of noise-induced oscillations in the FitzHugh-Nagumo system with $\tau$ at $D=0.09$ and different values of $K$ : (a) $K=0.1$ (b) $K=0.5$. Grey diamonds - peak periods $T_{i}, i=2,3, \ldots$, light-grey circles - basic period $T_{1}$.

without feedback as well, evolve with $K$ in a similar manner. An interesting observation is that at $K \approx 0.2$ a new peak appears with larger period, which did not exist without the feedback. As $K$ increases, both the period and the height of this peak grow monotonically until at $K \approx 1.75$ this peak becomes dominant. We observe that although the nature of oscillations in system (11) is different from that in (2), the behavior of the period of the spectrum peak induced by the feedback is qualitatively similar in both systems (compare Figs. $6(\mathrm{a})$ and $12(\mathrm{a}))$.

Hence, a phenomenon that could be called period entrainment by delayed feedback is realized here. The entraining effect of the delayed feedback on systems with two different kinds of noise-induced oscillations considered appears to be 

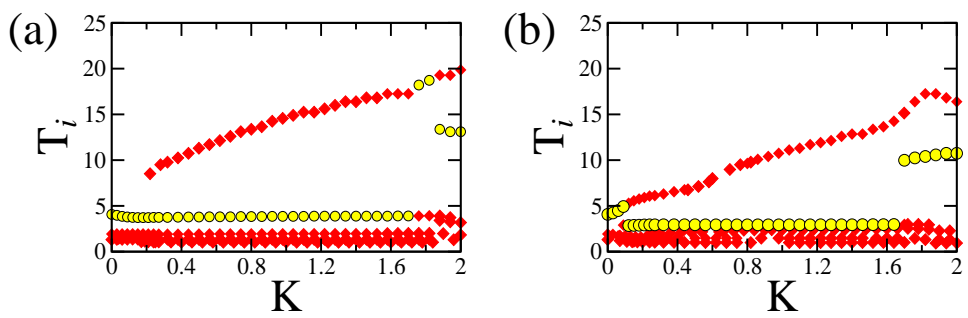

Fig. 12. Spectral peak periods $T_{i}, i=1,2, \ldots$ in the FitzHugh-Nagumo system for a range of $K$ at $D=0.09$ for two values of $\tau$ : (a) $\tau=4.12694 \approx T_{0}$, (b) $\tau=2.9$ Symbols are as in Fig. 11.

similar, the latter providing evidence for its generality.

At $\tau=2.9$ the period $T_{1}$ of the highest peak, i.e. $T_{0}$ without feedback, increases until at $K \approx 0.1$ the peak ceases to be dominant. However, it continues to exist and its period grows monotonically with $K$. At the same time several other peaks with smaller periods, which exist without feedback as well, hardly change their periods and heights. However, at $K \approx 0.1$ a new peak appears in the spectrum that becomes dominant as $K$ is increased a bit further. The period of this peak is close to $\tau$ and only slightly depends on $K$. The behavior of the period of this feedback-induced peak is qualitatively similar to the one in Eq. (2) (compare Figs. 6(b) and 12(b)). Thus, the basic period of oscillations is induced by feedback to coincide with $\tau$ within a proper range of $K$.

\section{Conclusions}

In the present work we propose a self-adaptive method for control of oscillations induced merely by noise. The method is based on using the delayed feedback loop in the form of the difference between the delayed and the current values of a signal measured from the system. The effectiveness of this control scheme is demonstrated for two different prototypes of coherence resonance oscillators, namely, a system near bifurcation, and a threshold system. As their examples, a Van der Pol oscillator below Andronov-Hopf bifurcation, and a simplified FitzHugh-Nagumo system were considered. For both systems it was shown that: (i) with a proper choice of feedback parameters $\tau$ and $K$ one can effectively control the coherence of noise induced oscillations in a desirable manner, i.e. improve or deteriorate the oscillation coherence deliberately; (ii) variation of time delay $\tau$ and/or feedback strength $K$ allows one to adjust the basic timescales of noise-induced motion; (iii) this adjustment can be realized either by entrainment of a period of the existing spectrum peak or by generation of a new spectral peak with period close to $\tau$.

In a system below Andronov-Hopf bifurcation, the control is due to the effect of the delayed feedback loop upon the stability properties of the fixed point 
in whose vicinity the noise-induced oscillations take place. In fact, delayed feedback gives rise to a countable set of eigenmodes, whose eigenperiods and stability are controlled by $\tau$. The highest peak in the spectrum of the noiseinduced motion is due to excitation of the most unstable mode. The coherence of oscillations is the higher, the less stable the mode is. With this, the control force appears to be minimal at those $\tau$ at which the coherence of controlled oscillations is maximal.

In a threshold excitable system the effect of a control loop is more complicated due to the nonlocal character of noise-induced motion. The latter includes two stages: oscillations around the fixed point and a long excursion in the phase space which provides return of the trajectory back to the vicinity of the fixed point. The delayed feedback influences either of these two stages in different ways, which leads to the difference between the value of $\tau$ at which the control force is minimal, and the value of $\tau$ at which oscillations are most coherent. With this, the force is minimal when delayed feedback makes the time intervals between successive excursions more regular.

\section{Acknowledgements}

AB and NJ acknowledge discussions with A. Nikitin. The work was supported by DFG in the framework of SFB555.

\section{References}

[1] T. Kapitaniak, Controlling Chaos: Theoretical and Practical Methods in NonLinear Dynamics, Academic Press (1996).

[2] H.G. Schuster, Handbook of Chaos Control: Foundations and Applications, Wiley-VCH (1999).

[3] S. Boccaletti, C. Grebogi, Y.-C. Lai, H. Mancini, and D. Maza, The control of chaos: theory and applications, Phys. Rep. 329 (2000) 103-197.

[4] R. Lima, M. Pettini, Suppression of chaos by resonant parametric perturbations, Phys. Rev. A 41 (1990) 726-733.

[5] E. Ott, C. Grebogi, and J. Yorke, Controlling chaos, Phys. Rev. Lett. 64 (1990) 1196-1199.

[6] K. Pyragas, Continuous control of chaos by self-controlling feedback, Phys. Lett. A 170 (1992) 421-428.

[7] R. Benzi, A. Sutera, and A. Vulpiani, The mechanism of stochastic resonance, J. Phys. A 14 (1981) L453-L458. 
[8] P. Jung, Periodically driven stochastic systems, Phys. Rep. 234 (1993) 175-295.

[9] Hu Gang, T. Ditzinger, C.Z. Ning, and H. Haken, Stochastic resonance without external periodic force, Phys. Rev. Lett. 71 (1993) 807-810.

[10] A.S. Pikovsky, J. Kurths, Coherence Resonance in a Noise-Driven Excitable System, Phys. Rev. Lett. 78 (1997) 775-778.

[11] K. Wiesenfeld, F.Moss, Stochastic resonance and the benefits of noise: from ice ages to crayfish and SQUID, Nature(London) 373 (1995) 33-36.

[12] V.S. Anishchenko, A.B. Neiman, F.Moss, and L. Schimansky-Geier, Stochastic resonance: noise enhanced order, Physics-Uspekhi 42 (1999) 7-39.

[13] G. Giacomelli, M. Giudici, S. Balle, and J.R. Tredicce, Experimental evidence of coherence resonance in an optical system, Phys. Rev. Lett.84 (2000) 3298-3301.

[14] K. Miyakawa, H. Isikawa, Experimental observation of coherence resonance in an excitable chemical reaction system, Phys. Rev. E 66 (2002) 046204.

[15] G. Schmidt, I. Goychuk, and P. Hänggi, Channel noise and synchronization in excitable membranes, Physica A 325 (2003) 165-175.

[16] V.V. Sherstnev, A. Krier, A.G. Balanov, N.B. Janson, A.N. Silchenko, and P.V.E. McClintock, The stochastic laser: mid-infrared lasing induced by noise, Fluct. and Noise Lett. 3 (2003) L91-L95.

[17] L. Gammaitoni, M. Locher, A. Bulsara, P. Hanggi, J. Neff, K. Wiesenfeld, W. Ditto, and M.E. Inchiosa, Controlling Stochastic Resonance. Phys. Rev. Lett. 82 (1999) 4574-4577.

[18] J.F. Lindner, J. Mason, J. Neff, B.J. Breen, W.L. Ditto, and A.R. Bulsara, Noninvasive control of stochastic resonance, Phys. Rev. E 63 (2001) 041107.

[19] D. Goldobin, M. Rosenblum, and A. Pikovsky, Controlling oscillator coherence by delayed feedback, Phys. Rev. E 67 (2003) 061119.

[20] P. S. Landa, A.A. Zaikin, M.G. Rosenblum, and J. Kurths, Control of noiseinduced oscillations of a pendulum with a randomly vibrating suspension axis, Phys. Rev. E 56 (1997) 1465-1470.

[21] N. Baba, A. Amann, E. Schöll, and W. Just, Giant improvement of time-delayed feedback control by spatio-temporal filtering, Phys. Rev. Lett. 89 (2002) 074101.

[22] O. Beck, A. Amann, E. Schöll, J.E.S. Socolar, and W. Just, Comparison of timedelayed feedback schemes for spatio-temporal control of chaos in a reactiondiffusion system with global coupling, Phys. Rev. E 66 (2002) 016213.

[23] J. Unkelbach, A. Amann, W. Just, and E. Schöll, Time-delay autosynchronization of the spatio-temporal dynamics in resonant tunneling diodes, Phys. Rev. E 68 (2003) 026204.

[24] W. Just, D. Reckwerth, J. Möckel, E. Reibold, and H. Benner, Delayed Feedback Control of Periodic Orbits in Autonomous Systems, Phys. Rev. Lett. 81 (1998) $562-565$. 
[25] S.K. Han, T.G. Yim, D.E. Postnov, and O.V. Sosnovtseva, Interacting Coherence Resonance Oscillators, Phys. Rev. Lett. 83 (1999) 1771-1774.

[26] H. Treutlein, K. Schulten, Noise-induced neural impulses, Eur. Biophys. J. 13 (1986) 355-365.

[27] By basic period we mean the inverse of the frequency of the highest peak in the Fourier power spectrum of oscillations. Given that the FPS normalized by the total energy of oscillations can be viewed as an energy distribution over frequencies, the basic period can be understood as the most probable period of oscillations. By mean period we mean the average time of phase trajectory return to some secant surface. These two periods coincide only in purely periodic oscillations.

[28] A. Neiman, P.I. Saparin, and L. Stone, Coherence resonance at noisy precursors of bifurcations in nonlinear dynamical systems, Phys. Rev. E 56 (1997) 270-273.

[29] A.G. Balanov, N.B. Janson, D.E. Postnov, and P.V.E. McClintock, Coherence resonance versus synchronization in a periodically forced self-sustained system, Phys. Rev. E 65 (2002) 041105.

[30] Time average of the control force $F$ is zero. Therefore, the variance $\left\langle F^{2}\right\rangle$ of $F$ is calculated as $F^{2}$ averaged over observation time.

[31] M. Scheutzow, Qualitative Behaviour of Stochastic Delay Equations with a Bounded Memory, Stochastics 12 (1984) 41-80.

[32] U. Küchler, B. Mensch, Langevin stochastic differential equation extended by a time delayed term, Stoch. Stoch. Rep. 40 (1992) 23-42.

[33] T. Ohira, Oscillatory correlation of delayed random walks, Phys. Rev. E 55 (1997) R1255-R1258.

[34] S. Guillouzic, I. L'Heureux, and A. Longtin, Small delay approximation of stochastic delay differential equations, Phys. Rev. E 59 (1999) 3970-3980.

[35] T. Ohira, T. Yamane, Delayed stochastic systems, Phys. Rev. E 61 (2000) 12471257.

[36] L.S. Tsimring, A. Pikovsky, Noise-Induced Dynamics in Bistable Systems with Delay, Phys. Rev. Lett. 87 (2001) 250602.

[37] C. Masoller, Noise-Induced Resonance in Delayed Feedback Systems, Phys. Rev. Lett. 88 (2002) 034102.

[38] B. Lindner, L. Schimansky-Geier, Analytical approach to the stochastic FitzHugh-Nagumo system and coherence resonance, Phys. Rev. E 60 (1999) $7270-7276$ 